\title{
Influencia del liderazgo directivo en el desempeño docente,
} Virú 2020

\section{Influence of directive leadership on teacher performance, Virú 2020}

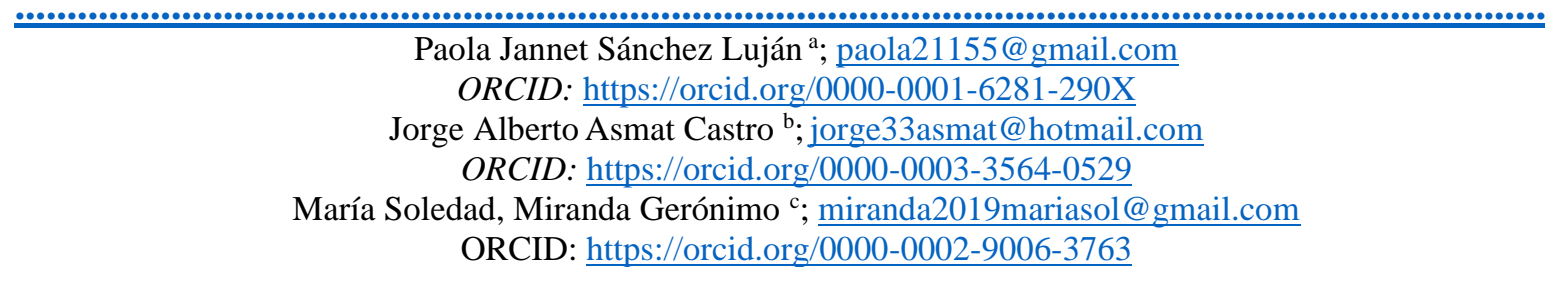

${ }^{a}$ Universidad Cesar Vallejo

${ }^{\mathrm{b}}$ Investigador Independiente

${ }^{\mathrm{c}}$ Universidad Cesar Vallejo

Recibido Octubre/ 19 / 2020 •Aceptado: Noviembre /24 / 2020 •Publicado: Diciembre / 29 / 2020

\section{RESUMEN}

La investigación tuvo como objetivo determinar la correlación entre el liderazgo directivo y el desempeño docente, Virú 2020. Además, proponer un modelo de liderazgo directivo para mejorar el desempeño docente en trabajo remoto. La investigación es de tipo no experimental, descriptiva y con diseño correlacional causal. Se aplicó un cuestionario de liderazgo directivo y una ficha de desempeño docente a una muestra de 81 docentes de educación secundaria seleccionados probabilísticamente. En los resultados se observó que el 58\% de docentes percibieron un nivel bajo de liderazgo, mientras que en el desempeño docente un 59\% se ubicó en nivel inicio. Así mismo, se obtuvo un valor de "rho" = 0,589 y un valor de significancia de 0,000 menor que el nivel de confiabilidad de 0,05. Concluyéndose que existe correlación entre ambas variables. Por tanto, a mayor nivel de liderazgo directivo mayor nivel de desempeño docente en los profesores de la UGEL de Virú 2020. Finalmente, se presentó como propuesta el modelo de liderazgo directivo para mejorar el desempeño docente en trabajo remoto cuya distinción es la sobrevaloración de tres características esenciales como: comunicación, confianza, y dominio digital.

Palabras clave: Liderazgo directivo- desempeño docente.

a Paola Jannet Sánchez Luján; ${ }^{\mathrm{b}}$ Jorge Alberto Asmat Castro y ${ }^{\mathrm{c}}$ María Soledad, Miranda Gerónimo Articulo Protegido por Licencia Creative Commons: BY-NC-ND / Protected by Creative Commons: BY-NC-ND.

Sendas es una revista de acceso abierto / Sendas is an Open Access Journal. 


\section{$\underline{\text { ABSTRACT }}$}

The present research aimed to determine the correlation between executive leadership and teaching performance, Virú 2020. In addition, to propose a management leadership model to improve teaching performance in remote work. The research is non-experimental, descriptive and with a causal correlational design. A managerial leadership questionnaire and a teacher performance sheet were applied to a sample of 81 probabilistically selected secondary school teachers. In the results, it was observed that $58 \%$ of teachers perceived a low level of leadership, while in teacher performance, $59 \%$ was at the beginning level. Likewise, a value of "rho" $=0.589$ and a significance value of 0.000 lower than the reliability level of 0.05 was obtained. Concluding that there is a correlation between both variables. Therefore, the higher the level of directive leadership, the higher the level of teaching performance in the teachers of the UGEL de Virú 2020. Finally, the managerial leadership model to improve teaching performance in remote work was presented as a proposal, the distinction of which is the overvaluation of three essential characteristics such as: communication, trust, and digital domain.

Keywords: managerial leadership- teacher performance.

\section{Introducción}

El liderazgo directivo, en los diferentes sistemas educativos, en estas dos últimas décadas ha sido considerado como uno de los factores más importantes después del desempeño docente para el logro de los aprendizajes de los estudiantes (Minedu, 2014; Waters, Marzano y McNulty,2003); en consecuencia, frente al desmesurado impacto negativo del Covid-
19, el rol del liderazgo debe adquirir una nueva forma de asumirlo. Interrogantes como: qué se debe y se puede aprender en estas condiciones, cómo orientar la acción docente y de las familias, para garantizar los aprendizajes de los estudiantes y el buen funcionamiento de la organización. Pues estas interrogantes deberán funcionalmente ser resueltas con gran acierto por el líder institucional. De no ser

\footnotetext{
${ }^{\mathrm{a}}$ Paola Jannet Sánchez Luján; ${ }^{\mathrm{b}}$ Jorge Alberto Asmat Castro y ${ }^{\mathrm{c}}$ María Soledad, Miranda Gerónimo Articulo Protegido por Licencia Creative Commons: BY-NC-ND / Protected by Creative Commons: BY-NC-ND. Sendas es una revista de acceso abierto / Sendas is an Open Access Journal.
} 
así, la crisis del aprendizaje de los estudiantes aumentará en gravedad. Si ya antes, 258 millones estudiantes en edad escolar estaban fuera de la escuela y la baja calidad educativa se traducía en un pobrísimo aprendizaje de lectura, matemática y ciencias. Y para finales de abril de 2020 se cerraron las escuelas en 180 países generando que un $80 \%$ no asista al colegio (Banco Mundial, 2020). Además, también se revela que son 165 millones de estudiantes perjudicados por la crisis de pandemia mundial (BID, 2020).

Con la suspensión y cierre obligado de las escuelas, los estudiantes vienen siendo perjudicados. En este marco contextual, la reacción no se ha hecho esperar y a nivel internacional diversos sistemas educativos han lanzado plataformas virtuales para ser trabajadas en modalidad remota. Así que el líder deberá asumir un comportamiento de acuerdo a las circunstancias para poder lograr que sus docentes se desempeñen óptimamente en un sistema de educación poco conocido y practicado. Es por eso que el comportamiento del liderazgo directivo debe ser observado en la situación actual el cual además de las cualidades de los otros estilos resulta ser la síntesis ecléctica de diferentes enfoques de liderazgo.

En Perú el liderazgo directivo, desde las políticas públicas, ha sido potenciado con la finalidad de mejorar el desempeño docente. No obstante, se presume que gran parte de los directivos, en el contexto actual, no están en condiciones de asumir el nuevo desafío. Ellos presentan dificultades en el dominio de competencias digitales básicas, las cuales son esenciales para desenvolverse eficientemente en una modalidad de trabajo remoto. Se suma a ello las escasa habilidades sociales y comunicativas, lo cual genera poca comunicación con sus docentes, escasa realización de trabajos colegiados, y un nulo o poquísimo ejercicio de acciones de monitoreo y acompañamiento. De modo que, estar conectado, generar confianza, trabajo colaborativo son comportamientos poco visibles en los directivos. Hecho que probablemente impacta negativamente en

\footnotetext{
${ }^{\mathrm{a}}$ Paola Jannet Sánchez Luján; ${ }^{\mathrm{b}}$ Jorge Alberto Asmat Castro y ${ }^{\mathrm{c}}$ María Soledad, Miranda Gerónimo Articulo Protegido por Licencia Creative Commons: BY-NC-ND / Protected by Creative Commons: BY-NC-ND. Sendas es una revista de acceso abierto / Sendas is an Open Access Journal.
} 
el desempeño docente en su momentánea modalidad.

Por tanto, es de vital importancia que las organizaciones educativas frente a estos nuevos desafíos trasciendan con nuevos modelos de liderazgo para desarrollar una educación de calidad que responda a los requerimientos y aspiraciones de Nuestro Proyecto Educativo Nacional 2021 en este contexto (Aldana, Salón y Guzmán, 2019). Esto sabiendo que el liderazgo históricamente ha funcionado como una solución a diversos problemas que han implicado la motivación de comportamientos sociales adecuados (Garfield, Rueden y Hagen, 2018). Al respecto, los peruanos Salvador y Sánchez (2018) sugieren que el sistema educativo se debe centrar en la formación de líderes eficientes para mejorar la gestión organizacional y quehacer pedagógica de los docentes.

Por otra parte, en la Ugel Virú, los docentes frente a la nueva situación han reaccionado con sorpresa y desconcierto. En esta nueva labor remota, se identifica que del $100 \%$ de estudiantes reportados en asuntos de conectividad, solo un $10 \%$ cuenta con una computadora y el servicio de internet. Otro grupo de $50 \%$ posee teléfono celular, no obstante, solo un $9 \%$ cuenta con internet, a esto se suma que no todos los equipos celulares de los estudiantes, un $30 \%$, son diseños digitales (videos, música, documentos, internet, whatsapp). Así a la falta de conectividad por internet $\mathrm{y}$ de tenencia de celulares sofisticados, se suma la ausencia de televisores en un $40 \%$ de los hogares de los estudiantes.

Como si fuera poco, el $80 \%$ de estudiantes que no cuenta con estos recursos tampoco cuenta con radio. Por otra parte, esta problemática no solo es de los estudiantes, también se manifiesta en el docente. Se identificó que un $55 \%$ de docentes no cuenta con computadora, y del grupo que lo tiene solo un $27 \%$ cuenta con internet fijo (Data, Ugel Virú marzo 2020). De las cifras se puede preguntar ¿Cuál son las acciones más acertadas que deben asumir los líderes directivos en este nuevo contexto para hacer que sus docentes puede desempeñarse eficientemente? ¿es

\footnotetext{
a Paola Jannet Sánchez Luján; ${ }^{\mathrm{b}}$ Jorge Alberto Asmat Castro y ${ }^{\mathrm{c}}$ María Soledad, Miranda Gerónimo Articulo Protegido por Licencia Creative Commons: BY-NC-ND / Protected by Creative Commons: BY-NC-ND. Sendas es una revista de acceso abierto / Sendas is an Open Access Journal.
} 
suficiente el modelo de liderazgo directivo que ellos practican para promover acciones que conlleven a alcanzar las metas de las instituciones educativas?

Siendo así, esta precariedad tenencia de recursos tecnológicos y también el bajo dominio de competencias digitales - ha calado en el actuar de los docentes causando efectos como: comportamiento desinteresado, actitud pesimista en la práctica pedagógica frente a la estrategia aprendo en casa $\mathrm{u}$ otra implementada por los colegios particulares.

Por lo expuesto anteriormente surge proponer un modelo de liderazgo directivo que resulte dinámico y útil en el nuevo contexto de educación y trabajo remoto que ha asumido el sistema educativo peruano por cuestiones coyunturales de la pandemia ocasionada por el COVID-19. Por ello en esta tesis se propone un modelo de liderazgo directivo para trabajo remoto, pretendiendo con ello direccionar la acción del director a generar en sus docentes un buen desempeño.
De ser así, entonces se puede prevenir un bajo nivel de desempeño docente y en consecuencia bajos niveles de rendimiento escolar frente a estas adversidades de largo alcance por solucionar. Caso contrario, se podría predecir graves consecuencias expresados en pobre resultados educativos, por debajo de lo que ya tenemos, por más esfuerzo e iniciativas de inversión en recursos tecnológicos y de conectividad, puesto que la investigación ha demostrado que factor fundamental para obtener buenos resultados escolares son las variables liderazgo directivo y desempeño docente. Por tanto, el modelo de liderazgo directivo para trabajo remoto se proyecta como alternativa, dentro de la coyuntura inesperada, a la actuación de un eficiente desempeño de los docentes de la UGEL de Virú.

Por último, metodológicamente aporta el estudio un nuevo instrumento adaptado para el tratamiento de la medición de la variable desempeño docente en trabajo remoto, el cual puede ser utilizado, adaptado y perfeccionado, en

${ }^{\mathrm{a}}$ Paola Jannet Sánchez Luján; ${ }^{\mathrm{b}}$ Jorge Alberto Asmat Castro y ${ }^{\mathrm{c}}$ María Soledad, Miranda Gerónimo Articulo Protegido por Licencia Creative Commons: BY-NC-ND / Protected by Creative Commons: BY-NC-ND. Sendas es una revista de acceso abierto / Sendas is an Open Access Journal. 
futuras investigaciones. Este instrumento ha sido acomodado a la situación actual de la labor docente en modalidad remota. Con estos dos últimos aportes metodológicos enfoque e instrumento, y los ya mencionados, se tiene el optimismo de que el rigor metodológico, la parsimonia y la ética elevarán el valor y aporte de la presente investigación en un contexto de práctica pedagógica abruptamente nueva.

Ahora bien, para direccionar la investigación se planteó el problema: ¿Cuál es la relación entre el liderazgo directivo y el desempeño docente de los profesores de la Ugel de Virú en el año 2020? También, el objetivo general determinar la correlación entre el liderazgo directivo y el desempeño docente de los profesores de la Ugel de Virú en el año 2020. Y las hipótesis: Existe correlación entre el liderazgo directivo y desempeño docente en las instituciones educativas de secundaria UGEL Virú 2020.

De manera general existe mucha investigación sobre el liderazgo en relación con las organizaciones educativas. No obstante, a nivel internacional más que como liderazgo directivo, gran parte de ella ha sido investigado refiriéndose a un enfoque o estilo determinado de liderazgo. A continuación, se procede a citar algunos antecedentes de interés para la presente tesis.

García (2019) en su tesis doctoral: Coordinación Pedagógica y Liderazgo distribuido en los Institutos de Secundaria. Universidad de Granada, tuvo como objetivo establecer la relación que existe entre la coordinación pedagógica y el liderazgo distribuido. El estudio concluye que el ejercicio de un cargo superior no demuestra la identidad de liderazgo ante los demás; que no solo es suficiente otorgar apoyo y orientar prácticas; sino, se requiere desarrollar un mayor nivel de formación en la que se orienta esta investigación.

Valle (2019) en su tesis doctoral El liderazgo distribuido y la percepción de los equipos directivos en las escuelas públicas de educación primaria madrileñas. Universidad Pablo de Olavide Sevilla; La Articulo Protegido por Licencia Creative Commons: BY-NC-ND / Protected by Creative Commons: BY-NC-ND Sendas es una revista de acceso abierto / Sendas is an Open Access Journal. 
investigación concluye la distribución del liderazgo en la mejora de resultados y de potenciar determinados contextos como las escuelas como elementos clave para el estudio.

Garay, (2019) en su tesis Liderazgo y logros en las organizaciones escolares de Chile, La investigación concluye que el proceso de influencia del liderazgo en la escuela es indirecto ya que puede haber ciertas variables mediadora como el esfuerzo extra, participación y satisfacción. Se afirma que el liderazgo de rango totales es más beneficioso, en el ámbito de la escuela, que el liderazgo transformacional.

El marco teórico que a continuación se desarrolla para el constructo liderazgo directivo, básicamente se puede ubicar dentro de dos enfoques epistemológicos: el primero desde un empirismo-analítico y el segundo desde un racionalismo-fenomenológico. Este marco epistemológico ayudará a comprender la validez de todo el conocimiento producido acerca de este constructo tratado explícita o implícitamente en las diferentes disciplinas humanas como la filosofía, psicología y sociología que más adelante se exponen.

No obstante, resulta ya axiomático decir que el liderazgo ontológicamente tiene existencia cuyo origen se remonta a los primeros grupos sociales de la humanidad, es decir, el líder como individuo real siempre ha existido como la figura conductora de un conjunto de individuos agrupados en organizaciones de diferentes niveles y clases que responden a los diferentes aspectos de la vida humana (familia, clan, comunidad, nación, empresa, ejércitos, etc.). Y, por otro lado, su capacidad de influencia sobre los individuos de un grupo u organización, capacidad que se manifiesta en conducir al grupo a la consecución de un determinado fin, sea de interés individual o colectivo.

En consecuencia, dos son las razones ontológicas que sustentan el constructo del líder como ente existente y objeto de estudio de las ciencias sociales

${ }^{\mathrm{a}}$ Paola Jannet Sánchez Luján; ${ }^{\mathrm{b}}$ Jorge Alberto Asmat Castro y ${ }^{\mathrm{C}}$ María Soledad, Miranda Gerónimo Articulo Protegido por Licencia Creative Commons: BY-NC-ND / Protected by Creative Commons: BY-NC-ND Sendas es una revista de acceso abierto / Sendas is an Open Access Journal. 
como un hecho social. La primera, la existencia de los grupos sociales; así sin organización y las relaciones que fluyen dentro de estas no hay líder. Y segundo, el líder queda definido por su cualidad innata de influencia sobre el grupo de individuos con él que se relaciona. En síntesis, ontológicamente se puede afirmar que alguien es líder si pertenece a una organización y mantiene relación de influencia sobre esta, generando diversos acontecimientos o conjuntos de hechos sociales posibles de ser estudiados objetiva o fenomenológicamente, según los enfoques epistemológicos que se consideren pertinentes (Tellez,1996; Padrón, 2014; Diaz y Camejo, 2015).

Volviendo a lo epistemológico-y si las pretensiones de muchos sistemas educativos es aspirar a la calidad educativa-entonces validar la aplicación y utilidad de conceptos como los de líder en el ámbito educativo es una necesidad que responde a los constantes cambios de la sociedad actual, y puntualmente que responda a la pregunta ¿en qué enfoques se sustenta el liderazgo directivo?

Desde el empirismo analítico el conocimiento acerca del liderazgo se considera válido en cuanto: primero este es un ente tangible posible de ser observado por la experiencia sensorial, aquí el positivismo se hace presente. Segundo, porque puede ser cuantificado y medido. Tercero, porque el conocimiento es verificable contrastable a la luz de los hechos, siendo que las hipótesis se convierten en leyes y teorías de regularidades constantes. A este paradigma, se alineo la psicología de la personalidad, específicamente la teoría de los rasgos, atribuyendo que el liderazgo es una cualidad innata que distingue a unos individuos de otros.

Por su parte, el racionalismo motivó a considerar un enfoque diferente en la obtención del conocimiento que consistió en prescindir de manera parcial del objeto como fuente primordial y asignarle a la razón la tarea de interpretar la realidad. Esta se sustenta en un

\footnotetext{
${ }^{\mathrm{a}}$ Paola Jannet Sánchez Luján; ${ }^{\mathrm{b}}$ Jorge Alberto Asmat Castro y ${ }^{\mathrm{c}}$ María Soledad, Miranda Gerónimo Articulo Protegido por Licencia Creative Commons: BY-NC-ND / Protected by Creative Commons: BY-NC-ND Sendas es una revista de acceso abierto / Sendas is an Open Access Journal.
} 
constructivismo que ontológicamente considera a la realidad como construcciones mentales y sociales. Así el liderazgo fue concebido como un fenómeno social producto de las relaciones sociales entre el líder y sus seguidores. Por tanto, no hay cualidades innatas, el liderazgo es una actividad que la puede ejercer cualquier individuo que se ejercite en aprender ciertas habilidades. Tributarios de este enfoque son modelos teóricos del liderazgo transformacional y distributivo (Tellez,1996; Padrón, 2014; Díaz y Camejo, 2015).

En el marco del buen desempeño docente no hay explícitamente una definición de lo que es desempeño docente; no obstante, se menciona qué implica un buen desempeño. Para ello considera un conjunto de dimensiones compartidas (colegiada, reflexiva, ética y relacional) y específicas (pedagógica, política y cultural) de la profesión docente. Es a partir de allí que se determinan cuatro dominios o campos en la que el profesor se desenvuelve profesionalmente y que si se cumplen cabalmente estos inciden favorablemente en el desarrollo integral de los estudiantes.

Por otra parte, para autores como Mateo (2000) el desempeño docente se refiere a la actuación docente en sus prácticas pedagógicas (planificación, conducción, evaluación y otros). Valdés (2004) lo relaciona con aspectos pedagógicos, emocional, responsabilidad funcional, relaciones interpersonales con los demás agentes y el resultado de los estudiantes. La OCDE (2001) lo identifica con los conocimientos del: contenido, estudiante, aprendizaje, motivación, recursos, tecnología, colaboración y capacidad reflexiva. Mientras que McBer (2000) con el profesionalismo, pensamiento, planificación, propuestas de metas, liderazgo y relación con los demás. Y Montenegro (2003) los define como conjunto de acciones concretas.

De estas ideas y teniendo como referencia principal el marco de buen desempeño docente se puede definir al desempeño docente como un conjunto de

${ }^{\mathrm{a}}$ Paola Jannet Sánchez Luján; ${ }^{\mathrm{b}}$ Jorge Alberto Asmat Castro y ${ }^{\mathrm{C}}$ María Soledad, Miranda Gerónimo Articulo Protegido por Licencia Creative Commons: BY-NC-ND / Protected by Creative Commons: BY-NC-ND. Sendas es una revista de acceso abierto / Sendas is an Open Access Journal. 
acciones concretas-basadas en dimensiones compartidas y específicasque posee y realiza el profesor en su labor diaria la cual implica continua reflexión teórica y práctica que permite innovar misiones pedagógicas para cumplir su rol. Esta práctica se centra en la enseñanza como un compromiso ético profesional para lograr el aprendizaje y desarrollo integral del estudiante, de modo que el desempeño está relacionado con: la preparación para el aprendizaje de los estudiantes y enseñanza para el aprendizaje de los estudiantes, participación en la gestión de la escuela articulada con la comunidad, y, desarrollo de la profesionalidad y la identidad docente (Minedu, 2014). Se advierte que para fines de este estudio y por cuestiones de condiciones remotas solo se toman las dos primeras.

\section{Metodología}

Todo estudio se enmarca en un determinado enfoque de investigación. El presente, sigue un enfoque cuantitativo el cual se puede caracterizar como aquel que mide fenómenos, utiliza estadísticas, contrasta hipótesis y efectúa análisis de causa efecto. Además, este se construye de manera secuencial y es probatorio.

En cuanto al diseño de investigación se asume en el presente estudio, de acuerdo Sánchez y Reyes (1996) un diseño correlacional. Aquí las variables no fueron controladas, es de carácter descriptiva porque parte del recojo de información de las variables en su situación actual y porque se pretende encontrar una relación estadística significativa de causa efecto (Hernández y Col. 2010).

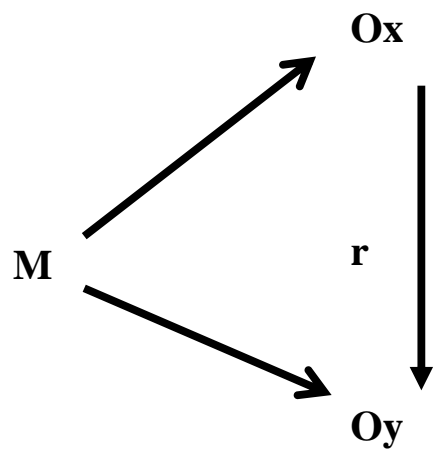

Leyenda:

$\mathrm{M}=$ Muestra observada (docentes de instituciones educativas públicas provincia de Virú).

${ }^{\mathrm{a}}$ Paola Jannet Sánchez Luján; ${ }^{\mathrm{b}}$ Jorge Alberto Asmat Castro y ${ }^{\mathrm{c}}$ María Soledad, Miranda Gerónimo Articulo Protegido por Licencia Creative Commons: BY-NC-ND / Protected by Creative Commons: BY-NC-ND. Sendas es una revista de acceso abierto / Sendas is an Open Access Journal. 
$\mathrm{r}=\quad$ Relación causal entre las variables

La población del presente estudio estudiadas.

estuvo constituida por todos los docentes,

$\mathrm{Ox}=$ Observación de la variable de ambos sexos, pertenecientes a las relacional1: (Liderazgo directivo).

Oy = Observación de la variable relaciona instituciones educativas nivel secundario 12: (desempeño docente). de la Ugel Virú 2020. Además, su condición laboral es de contratados y nombrados.

Tabla 1: Distribución de la población de docentes de las instituciones educativas nivel secundario Ugel Virú, 2020

\begin{tabular}{llllllll}
\hline \multirow{2}{*}{$\begin{array}{l}\text { Cantidad de } \\
\text { Instituciones educativas }\end{array}$} & \multicolumn{3}{l}{ Docentes sexo } & \multicolumn{3}{l}{ Total } \\
\cline { 2 - 7 } & \multicolumn{2}{l}{ Masculino } & \multicolumn{2}{l}{ Femenino } & & \\
\cline { 2 - 7 } & $\mathrm{N}$ & $\%$ & $\mathrm{~N}$ & $\%$ & $\mathrm{~N}$ & $\%$ \\
\hline 27 & 213 & 49 & 218 & 51 & 431 & 100 \\
\hline
\end{tabular}

Nota: Nóminas de docentes base de datos Ugel Virú 2020

La muestra estuvo representada por el $20 \%$, que son 86 docentes de ambos sexos, contratados y nombrados, pertenecientes al nivel de secundaria de educación básica de la población total, cuyas características presentaron similitud homogénea y la unidad de la muestra la constituyó cada uno de los docentes seleccionados. La presente investigación es una producción textual original. De modo que todo lo redactado en sus diferentes partes constituyen información procesada por la autora a partir de las fuentes bibliográficas referenciadas $\mathrm{y}$

a Paola Jannet Sánchez Luján; ${ }^{\mathrm{b}}$ Jorge Alberto Asmat Castro y ${ }^{\mathrm{c}}$ María Soledad, Miranda Gerónimo Articulo Protegido por Licencia Creative Commons: BY-NC-ND / Protected by Creative Commons: BY-NC-ND. Sendas es una revista de acceso abierto / Sendas is an Open Access Journal. 
Sendas. 1(1), Octubre - Diciembre, 2020.

URL: https://www.revistas.infoc.edu.pe/index.php/sendas

Email: revistasendas@infoc.edu.pe

citadas aquí. Con ello la tesis

alcanza su dimensión ética

esencial, y evita así caer en el

plagio y autoplagio que daña todo

crédito de un profesional, por más

eficiente que sea. También, se
Vol. 1, $\mathrm{N}^{\circ} 4$, pp. 38 - 64 Diciembre 2020

ISSN $2708-6380$ https://doi.org/10.47192/rcs.v1i4.49

evidencia el respeto por la propiedad intelectual, es decir las ideas de otros investigadores. Esto se hace mencionando las referencias bibliográficas respectivas.

\section{$\underline{\text { Resultados }}$}

Tabla 2:

Nivel general de liderazgo directivo según profesores de las instituciones educativas de la UGEL Virú, 2020

\begin{tabular}{ccc}
\hline Nivel & \multicolumn{2}{c}{ Liderazgo directivo } \\
\cline { 2 - 3 } & F & $\%$ \\
\hline Alto & 9 & 11 \\
\hline Medio & 25 & 31 \\
\hline Bajo & 47 & 58 \\
\hline Total & 81 & 100 \\
\hline
\end{tabular}

Nota: Matriz de puntaje brutos de liderazgo directivo

En la tabla 2, se observa que 9 docentes que representan el $11 \%$ de un total de 81 , percibieron un nivel alto de liderazgo directivo. Otros 25 que representan el $31 \%$ percibieron un nivel medio. Mientras que 47 docentes que representan el $58 \%$ lo percibieron en un nivel bajo.

a Paola Jannet Sánchez Luján; ${ }^{\mathrm{b}}$ Jorge Alberto Asmat Castro y ${ }^{\mathrm{c}}$ María Soledad, Miranda Gerónimo Articulo Protegido por Licencia Creative Commons: BY-NC-ND / Protected by Creative Commons: BY-NC-ND. Sendas es una revista de acceso abierto / Sendas is an Open Access Journal. 
Sendas. $1(1)$, Octubre - Diciembre, 2020.

Vol. 1, $\mathrm{N}^{\circ} 4$, pp. 38 - 64 Diciembre 2020

URL: https://www.revistas.infoc.edu.pe/index.php/sendas

Email: revistasendas@infoc.edu.pe

\section{Tabla 3}

Nivel de liderazgo directivo, por dimensiones, según profesores de las instituciones educativas de la UGEL Virú, 2020

\begin{tabular}{|c|c|c|c|c|c|c|c|c|}
\hline \multirow[t]{2}{*}{ Nivel } & \multicolumn{2}{|c|}{$\begin{array}{l}\text { Dirección } \\
\text { de futuro }\end{array}$} & \multicolumn{2}{|c|}{$\begin{array}{c}\text { Desarrollo } \\
\text { personal }\end{array}$} & \multicolumn{2}{|c|}{$\begin{array}{c}\text { Rediseño } \\
\text { de la organización }\end{array}$} & \multicolumn{2}{|c|}{$\begin{array}{c}\text { Gestionar } \\
\text { la Instrucción }\end{array}$} \\
\hline & $\mathbf{f}$ & $\%$ & f & $\%$ & f & $\%$ & $\mathbf{f}$ & $\%$ \\
\hline Alto & 13 & 16 & 8 & 10 & 9 & 11 & 10 & 12 \\
\hline Medio & 14 & 17 & 29 & 36 & 16 & 20 & 66 & 81 \\
\hline Bajo & 54 & 67 & 44 & 54 & 56 & 69 & 5 & 6 \\
\hline Total & 81 & 100 & 81 & 100 & 81 & 100 & 81 & 100 \\
\hline
\end{tabular}

Nota: Matriz de puntaje brutos de liderazgo directivo

En la tabla 3, se observa los niveles del liderazgo directivo por dimensiones. La dimensión dirección de futuro fue percibida por el $16 \%$ de docentes en un nivel alto. Otro $17 \%$ lo percibió en nivel medio, y un $67 \%$ lo percibió en nivel bajo. La dimensión desarrollo personal fue percibida por un $10 \%$ de docentes en un nivel alto. Otro $36 \%$ lo percibió en un nivel medio. Y un $54 \%$ de docentes la percibió en un nivel bajo. La dimensión rediseño de la organización fue percibida por un $11 \%$ de docentes en un nivel alto. Otro $20 \%$ lo percibió en un nivel medio. Y un $69 \%$ de docentes la percibió en un nivel bajo. La dimensión gestionar la instrucción fue percibida por un $12 \%$ de docentes en un nivel alto. Otro $81 \%$ lo percibió en un nivel medio. Mientras que un $6 \%$ de docentes la percibió en un nivel bajo.

${ }^{\mathrm{a}}$ Paola Jannet Sánchez Luján; ${ }^{\mathrm{b}}$ Jorge Alberto Asmat Castro y ${ }^{\mathrm{c}}$ María Soledad, Miranda Gerónimo Articulo Protegido por Licencia Creative Commons: BY-NC-ND / Protected by Creative Commons: BY-NC-ND. Sendas es una revista de acceso abierto / Sendas is an Open Access Journal. 


\section{Tabla 4}

Nivel general de desempeño docente de los profesores de las instituciones educativas de secundaria UGEL Virú, 2020

\begin{tabular}{ccc}
\cline { 2 - 3 } Nivel & \multicolumn{2}{c}{ Desempeño docente } \\
\cline { 2 - 3 } & $\boldsymbol{f}$ & $\boldsymbol{\%}$ \\
\hline Logrado & 14 & 17 \\
\hline Proceso & 19 & 23 \\
\hline Inicio & 48 & 59 \\
\hline Total & 81 & 100 \\
\hline
\end{tabular}

Nota: Matriz de puntaje brutos de desempeño docente

En la tabla 4, se observa que

14 profesores que representan el

$17 \%$ de un total de 81 , se ubicaron

en un nivel logrado de desempeño

docente. Otros 19 que representan el $23 \%$ alcanzaron un nivel

proceso. Mientras que 48 profesores que representan el $59 \%$ se ubicaron en el nivel inicio.

${ }^{\mathrm{a}}$ Paola Jannet Sánchez Luján; ${ }^{\mathrm{b}}$ Jorge Alberto Asmat Castro y ${ }^{\mathrm{c}}$ María Soledad, Miranda Gerónimo Articulo Protegido por Licencia Creative Commons: BY-NC-ND / Protected by Creative Commons: BY-NC-ND. Sendas es una revista de acceso abierto / Sendas is an Open Access Journal. 


\section{Tabla 5}

Nivel de desempeño docente, por dimensiones, de los profesores de las instituciones educativas de secundaria UGEL Virú, 2020

\begin{tabular}{ccccc} 
& \multicolumn{2}{c}{$\begin{array}{c}\text { Preparación Aprendizaje } \\
\text { Enseñanza Aprendizaje }\end{array}$} \\
& \multicolumn{2}{c}{ Estudiantes } & \multicolumn{2}{c}{ Estudiante } \\
\cline { 2 - 5 } & $\boldsymbol{f}$ & $\boldsymbol{F}$ & $\boldsymbol{F}$ \\
\hline Logrado & 20 & 25 & 13 & 16 \\
\hline Proceso & 39 & 48 & 20 & 25 \\
\hline Inicio & 22 & 27 & 48 & 100 \\
\hline Total & 81 & 100 & 81 &
\end{tabular}

Nota: Matriz de puntaje brutos de desempeño docente

En la tabla 5, se observa los niveles del desempeño docente por dimensiones. En la dimensión preparación para el aprendizaje de los estudiantes el $25 \%$ de docentes se ubicó en el nivel logrado. Otro grupo de un $48 \%$ de profesores se ubicó en el nivel proceso; mientras que el $27 \%$ se ubicó en el nivel inicio. En la dimensión enseñanza para el aprendizaje de los estudiantes el $16 \%$ de docentes se ubicó en el nivel logrado. Otro grupo de un $25 \%$ de profesores se ubicó en el nivel proceso; mientras que el $59 \%$ se ubicó en el nivel inicio.

${ }^{\mathrm{a}}$ Paola Jannet Sánchez Luján; ${ }^{\mathrm{b}}$ Jorge Alberto Asmat Castro y ${ }^{\mathrm{c}}$ María Soledad, Miranda Gerónimo Articulo Protegido por Licencia Creative Commons: BY-NC-ND / Protected by Creative Commons: BY-NC-ND. Sendas es una revista de acceso abierto / Sendas is an Open Access Journal. 


\section{Tabla 18}

\section{Pruebas de contrastación de hipótesis}

Correlación entre las variables liderazgo directivo y desempeño docente en las instituciones educativas de secundaria UGEL Virú 2020

\begin{tabular}{|c|c|c|c|c|}
\hline \multicolumn{3}{|c|}{ HIPÓTESIS } & \multicolumn{2}{|c|}{ Regla de decisión } \\
\hline$H_{0}=$ & \multicolumn{2}{|c|}{$\begin{array}{l}\text { No existe correlación entre el liderazgo directivo y } \\
\text { desempeño docente en las instituciones educativas de } \\
\text { secundaria UGEL Virú } 2020\end{array}$} & $\begin{array}{l}\text { Si } p \geq \\
0,05\end{array}$ & $\begin{array}{l}\text { Se acepta } \\
\text { hipótesis } \\
\text { nula }\end{array}$ \\
\hline$H a=$ & \multicolumn{2}{|c|}{$\begin{array}{l}\text { Existe correlación entre el liderazgo directivo y } \\
\text { desempeño docente en las instituciones educativas de } \\
\text { secundaria UGEL Virú } 2020\end{array}$} & $\begin{array}{l}\text { Si } \mathrm{p} \leq \\
0,05\end{array}$ & $\begin{array}{l}\text { Se rechaza } \\
\text { hipótesis } \\
\text { nula }\end{array}$ \\
\hline & & & $\begin{array}{l}\text { Desempeño } \\
\text { docente }\end{array}$ & $\begin{array}{l}\text { Liderazgo } \\
\text { directivo }\end{array}$ \\
\hline \multirow{6}{*}{$\begin{array}{l}\text { Rho de } \\
\text { Spearman }\end{array}$} & \multirow[t]{3}{*}{ Desempeño docente } & $\begin{array}{l}\text { Coeficiente de } \\
\text { correlación }\end{array}$ & 1,000 &, $588^{* *}$ \\
\hline & & Sig. (bilateral) & & $\overline{0}, 000$ \\
\hline & & $\mathrm{N}$ & 81 & 81 \\
\hline & Liderazgo directivo & $\begin{array}{l}\text { Coeficiente de } \\
\text { correlación }\end{array}$ &, $588^{* *}$ & 1,000 \\
\hline & & Sig. (bilateral) & ,000 & \\
\hline & & $\mathrm{N}$ & 81 & 81 \\
\hline
\end{tabular}

**. La correlación es significativa en el nivel 0,01 (bilateral).

En la tabla 6 se observa que el valor de la significancia bilateral $=0,00$ es menor que el nivel de significancia de 0,05. De tal modo se concluye que existe

a Paola Jannet Sánchez Luján; ${ }^{\mathrm{b}}$ Jorge Alberto Asmat Castro y ${ }^{\mathrm{c}}$ María Soledad, Miranda Gerónimo Articulo Protegido por Licencia Creative Commons: BY-NC-ND / Protected by Creative Commons: BY-NC-ND. Sendas es una revista de acceso abierto / Sendas is an Open Access Journal. 
una relación estadística significativa entre el liderazgo directivo y desempeño docente en las instituciones educativas de secundaria UGEL Virú 2020. También el coeficiente $\mathrm{rho}=0,588$ indica que la correlación es positiva y moderada. Por tanto, a mayor nivel de liderazgo directivo mayor nivel de desempeño docente en los profesores de la UGEL de Virú.

\section{Discusión}

El propósito fundamental de la presente investigación —más allá de reafirmar la importancia del liderazgo directivo en la funcionalidad de la escuela y el logro de los aprendizajes de los estudiantes, puntualmente aquí en su influencia en el desempeño docente, tal y como lo han sostenido diversas investigaciones (Fischman 2017; Handy 1997; Leithwood,2009) - ha sido advertir de la necesidad de una nueva práctica de liderazgo directivo para una modalidad de educación a distancia en contexto virtual el cual de aquí para los años venideros se presenta como una cuestión obligada.
Aunque, ha sido ampliamente investigada la mencionada relación aportando evidencia favorable. No obstante, amerita estar en reflexión permanente, mucho más en nuevo contexto educativo impactado pavorosamente por el virus asolador covid-19, obligando a la mayoría de sistemas educativos en el mundo a cerrar sus escuelas y asumir una modalidad de educación no presencial a distancia, es decir remota. Perú no ha sido ajeno a esta medida de emergencia de sanidad mundial. En este marco se esfuerza la presente investigación por observar, describir y explicar la influencia del liderazgo directivo en el desempeño de los docentes. Para ello, se formuló el objetivo de correlacionar las variables liderazgo directivo y desempeño docente.

Los resultados expuestos identifican que un $58 \%$ de docentes de las instituciones educativas de la Ugel de Virú perciben un nivel bajo de liderazgo (Tabla 6). Así mismo, en el desempeño docente se ha observado que un $59 \%$ de docentes ha

${ }^{\mathrm{a}}$ Paola Jannet Sánchez Luján; ${ }^{\mathrm{b}}$ Jorge Alberto Asmat Castro y ${ }^{\mathrm{c}}$ María Soledad, Miranda Gerónimo Articulo Protegido por Licencia Creative Commons: BY-NC-ND / Protected by Creative Commons: BY-NC-ND. Sendas es una revista de acceso abierto / Sendas is an Open Access Journal. 
ocupado un nivel bajo (Tabla 8). Estas cifras que señalan niveles bajos, a diferencia de algunos antecedentes citados aquí cuyos datos muestran que los niveles alcanzados de ambas variables son regular y altos (De La Cruz,2018; Zúñiga, 2018; Luque, 2018; Ordinola, 2019). Esta diferencia, en primer lugar, pueda que se deba a sesgos de carácter metodológicos. Por ejemplo, el tamaño de las muestras en todas estas investigaciones fue menor a 50 sujetos.

Ahora bien, también, podría atribuirse presumiblemente, a la nueva modalidad educativa. Así resulta convencionalmente más fácil y cómodo en modalidad presencial ejercer y ejecutar con mayor eficacia actividades de liderazgo como: dirección de futuro (comunicación con los docentes para compartir la visión, misión y objetivos de la institución. También la sensibilización de los docentes para asumir compromisos, generar expectativas para lograr metas). Lo mismo sucede con actividades relacionadas con el desarrollo del personal institucional (actualizaciones, delegación de funciones, confianza en el monitoreo y acompañamiento).

Los resultados referidos a la correlación entre las variables estudiadas, liderazgo directivo y desempeño docente, de manera general han arrojado un valor $r h o=0,589$ y una significancia bilateral $=$ 0,000 menor que 0,05 (Tabla: 19). Estos datos indican que entre las variables existe una correlación positiva, moderada y significativa. Es decir que a mayor liderazgo directivo mayor nivel de desempeño docente. Con esto se rechaza la hipótesis nula y se acepta la de investigación alterna.

Estos resultados concuerdan con algunos de los antecedentes citados (García, 2019; De La Cruz, 2018; Luque,2018; Ordinola, 2019) quienes encontraron en sus investigaciones también una correlación positiva y moderada. Bajo estos resultados, se interpreta que el liderazgo directivo es fundamental para lograr una gestión exitosa de la organización educativa

${ }^{\mathrm{a}}$ Paola Jannet Sánchez Luján; ${ }^{\mathrm{b}}$ Jorge Alberto Asmat Castro y ${ }^{\mathrm{c}}$ María Soledad, Miranda Gerónimo Articulo Protegido por Licencia Creative Commons: BY-NC-ND / Protected by Creative Commons: BY-NC-ND. Sendas es una revista de acceso abierto / Sendas is an Open Access Journal. 
(Bolívar, 2010). Las investigaciones citadas concuerdan que acciones como: el logro de la visión y misión, el desarrollo personal, la articulación de la gestión comunal, el acompañamiento, monitoreo están asociadas a buenas prácticas pedagógicas relacionados con los aprendizajes de los estudiantes.

No obstante, hay resultados como los de Zuñiga (2018) cuyos resultados arrojaron una correlación negativa y poco significativa entre las variables, por lo que el autor sostiene que es poco probable que el desempeño docente esté asociado al liderazgo directivo, y más bien sea influenciado por otras variables. Afirmación muy discutible, puesto que, a nivel descriptivos, en este estudio, ambas variables se identifican con niveles medios en proporciones mayores al 50\% de equivalentes. Nuevamente, se puede argumentar que estos resultados sean sesgos metodológicos del mismo tipo indicados arriba.

Además, el hecho que el directivo de la escuela no cuente con las condiciones o cualidades de un líder. Con esto último quedaría demostrado que la teoría de los rasgos (Cattell, 1973) es insuficiente para afirmar que los líderes son personajes exclusivamente nacidos para tal fin. $\mathrm{Al}$ respecto, más allá de las posturas filosóficas, Elmore (2008) — citado en el marco teórico - sostiene que la condición del líder escolar es su capacidad de mejorar la práctica docente.

Por otra parte, los resultados permiten aportar evidencia a las diferentes teorías del liderazgo expuestas en el marco teórico. Ontológicamente se corrobora la existencia del líder como un ente y hecho social, cuyo campo de acción es la organización en donde su cualidad esencial es la capacidad de influencia capaz de movilizar al conjunto de agentes sociales pertenecientes a una organización hacia una determinada meta o fin.

En la dimensión dirección del futuro relacionada con el desempeño se obtuvo una rho $=0,592$ y una significancia bilateral de 0,000. Se halla en estos resultados una correlación positiva,

\footnotetext{
${ }^{\mathrm{a}}$ Paola Jannet Sánchez Luján; ${ }^{\mathrm{b}}$ Jorge Alberto Asmat Castro y ${ }^{\mathrm{c}}$ María Soledad, Miranda Gerónimo Articulo Protegido por Licencia Creative Commons: BY-NC-ND / Protected by Creative Commons: BY-NC-ND Sendas es una revista de acceso abierto / Sendas is an Open Access Journal.
} 
moderada y significativa. Se interpreta con ellos que todo líder con capacidad de transmitir la visión y misión-en ellas las metas del largo alcance de la institución educativa - será capaz de lograr que los demás agentes de la organización compartan objetivos y metas comunes. Dentro de las teorías, y particularmente Leithwood (2009), ha señalado que esta dimensión es fundamental para el éxito organizacional y que para ello hay que tener mucho poder comunicativo y de persuasión para lograr alinear a todos los agentes en una visión común respecto al éxito de la escuela.

Aunque, tal como lo señala en su investigación Garay (2019) que estos objetivos son muchos más posibles en las escuelas con un tipo de liderazgo que va más allá que los otros tipos (Transaccional, transformacional y distributivo), o sea un liderazgo de rango total. Es muy presumible que el liderazgo aplicado en la Ugel de Virú no considere ninguno de los casos debido que la ausencia de presencialidad no le permite desenvolverse tal y como lo hacía en una modalidad de educación presencial.

Este vacío de liderazgo se ha visto expresado en aspectos del desempeño docente como los de no sentirse vinculados con la institución y sus agentes, dado que un buen número de profesores no conoce la visión y misión, ni mucho menos las condiciones reales de las características socioeconómicas y geográficas de los estudiantes; tampoco han percibido que se les haya brindado oportunidades de organizar la escuela en una nueva situación, hecho que ha generado en los docentes escaza motivación y bajas expectativas respecto a su desempeño docente.

Estás ideas quedan corroboradas con De La Cruz (2018) quien estudiando las mismas dimensiones llega a ideas similares. Afirma que un buen líder directivo es aquel que logra hacer transcender a la organización educativa mediante el alance de la misión y visión.

La correlación obtenida entre la dimensión desarrollo personal y

\footnotetext{
${ }^{\mathrm{a}}$ Paola Jannet Sánchez Luján; ${ }^{\mathrm{b}}$ Jorge Alberto Asmat Castro y ${ }^{\mathrm{c}}$ María Soledad, Miranda Gerónimo Articulo Protegido por Licencia Creative Commons: BY-NC-ND / Protected by Creative Commons: BY-NC-ND Sendas es una revista de acceso abierto / Sendas is an Open Access Journal.
} 
desempeño docente cuyo valor fue de rho $=0,577$, permite interpretar que los docentes de la Ugel de Virú sienten poco apoyo por parte de su directivo. Esto se evidencia en el desconocimiento que tienen los profesores en cuanto a la estrategia virtual aprendo en casa (propuesta del Minedu frente a la emergencia nacional), así mismo en el poco dominio del uso de plataformas virtuales; las ausencias de trabajos colegiados, la no generación de confianza y de un clima institucional; la desconfianza para delegar funciones. No concuerda la investigación con otros autores (De La Cruz,2018; Luque, 2018; Ordinola, 2019) quienes sostienen que una de las actividades más recurrentes del directivo es la realización de capacitaciones para sus docentes.

No obstante, tal vez ahora, a los directivos ya sea por falta de dominio de competencias en el uso de herramientas tecnológicas se les haga más difícil que antes ejecutar estas actividades, a diferencia de cuando las hacían en modalidad presencial. Por otra parte, es de destacar que Valle (2019) y García (2019) han señalado que un liderazgo distributivo permite ser efectivo en todo lo relacionado con el desarrollo personal. Sin embargo, se propone que para un escenario de educación en contexto de trabajo remoto vaya más allá de un simple liderazgo distributivo.

Así mismo, la correlación de rho $=0,566$ entre el rediseño organizacional con el desempeño docente permite interpretar, que el desajuste por ausencia de presencialidad ha impactado negativamente en el liderazgo directivo, y en consecuencia en el desempeño docente a los profesores de la Ugel de Virú.

En lo que respecta al coeficiente de $r h o=0,517$ obtenido de la correlación entre la gestión de la instrucción y el desempeño docente hace ver que efectivamente la correlación hallada es positiva, moderada y significativa. A estas alturas del desarrollo de la discusión, se puede afirmar ya que las correlaciones mencionadas y esta son de orden causal.

a Paola Jannet Sánchez Luján; ${ }^{\mathrm{b}}$ Jorge Alberto Asmat Castro y ${ }^{\mathrm{c}}$ María Soledad, Miranda Gerónimo Articulo Protegido por Licencia Creative Commons: BY-NC-ND / Protected by Creative Commons: BY-NC-ND. Sendas es una revista de acceso abierto / Sendas is an Open Access Journal. 
Dado que la investigación ha sido consistente acerca del liderazgo en los diferentes aspectos de la organización educativa (clima institucional, gestión comunal, administrativa e institucional). Últimamente en los resultados de los aprendizajes de los estudiantes, claro está mediados por el desempeño docente (Marzano, 2005; Robinson, 2007). Con todo, los resultados evidenciaron que los líderes de la Ugel Virú no han logrado mejorar estos aspectos de la organización, dado que los resultados de desempeño docente evidenciaron que los profesores no han recibido acompañamiento, monitoreo y orientaciones pedagógicas.

Se ha expuesto y discutido los resultados en relación con otros hallazgos en las investigaciones citadas aquí. De todo, y considerando las evidencias presentadas en los antecedentes y el marco teórico se puede concluir que la correlación entre las variables liderazgo directivo y desempeño docente es causal. Aunque, pueda ser mucho presumir para otros investigadores, dado que generalmente los estudios correlacionales son de orden estadístico; no obstante, la evidencia empírica permite afirmar grado de causalidad.

Por otra parte, se sostuvo que frente al contexto actual - trabajo remoto - urge la necesidad de proponer un tipo de liderazgo de acuerdo a las circunstancias, cuyas características logren impactar positivamente en el desempeño docente. Lo último a razón de que se considera que es insuficiente los diferentes enfoques de liderazgos expuestos.

Finalmente, sobre estas dos posturas se deja abierto el campo a la investigación para todos aquellos que deseen contrastar las ideas expuestas sobre la relación del liderazgo directivo y desempeño docente, así como la necesidad de una propuesta de un nuevo líder que permita superar las barreras de la comunicación, el tiempo y espacio físico interpuestas obligadamente por un mundo virtual que de aquí en adelante será cosa cotidiana en la labor educativa de todo docente y directivo.

${ }^{\mathrm{a}}$ Paola Jannet Sánchez Luján; ${ }^{\mathrm{b}}$ Jorge Alberto Asmat Castro y ${ }^{\mathrm{c}}$ María Soledad, Miranda Gerónimo Articulo Protegido por Licencia Creative Commons: BY-NC-ND / Protected by Creative Commons: BY-NC-ND. Sendas es una revista de acceso abierto / Sendas is an Open Access Journal. 


\section{Conclusiones}

1. Se determinó que existe una relación positiva, moderada y estadísticamente significativa entre el liderazgo directivo y desempeño docente en las instituciones educativas de secundaria UGEL Virú 2020. Dado que el valor del coeficiente rho $=0,588$. Además, el valor de la significancia bilateral $=$ 0,00 es menor que el nivel de significancia de 0,05 . Por tanto, a mayor nivel de liderazgo directivo mayor nivel de desempeño docente en los profesores de la UGEL de Virú.

2. Se identificó que el liderazgo directivo que perciben los docentes presenta un nivel bajo. Dado que un $58 \%$ lo calificó en nivel bajo; un 31\% en nivel medio, mientras que solo un $11 \%$ lo concibió en un nivel alto.

3. Se identificó que el desempeño docente fue calificado en un nivel inicio. Debido a que las cifras porcentuales revelan que un $59 \%$ fue calificado en nivel inicio; otro $23 \%$ fue calificado en nivel proceso, mientras que solo un $17 \%$ se calificó en un nivel logrado.

4. Se determinó que existe una relación positiva, moderada y estadísticamente significativa entre la dirección de futuro y el desempeño docente en las instituciones educativas de secundaria UGEL Virú 2020. Dado que el valor del coeficiente rho=0,592. Además, el valor de la significancia bilateral $=$ 0,00 es menor que el nivel de significancia de 0,05 . Por tanto, a mayor nivel de dirección de futuro mayor nivel de desempeño docente.

5. Se determinó que existe una relación positiva, moderada y estadísticamente significativa entre el desarrollo personal y el desempeño docente en las instituciones educativas de secundaria UGEL Virú 2020. Dado que el valor del coeficiente rho= 0,577. Además, el valor de la significancia bilateral $=0,00$ es menor que el nivel de significancia de 0,05 . Por tanto, a mayor nivel de desarrollo personal mayor nivel de desempeño docente.

a Paola Jannet Sánchez Luján; ${ }^{\mathrm{b}}$ Jorge Alberto Asmat Castro y ${ }^{\mathrm{c}}$ María Soledad, Miranda Gerónimo Articulo Protegido por Licencia Creative Commons: BY-NC-ND / Protected by Creative Commons: BY-NC-ND. Sendas es una revista de acceso abierto / Sendas is an Open Access Journal. 
6. Se determinó que existe una relación positiva, moderada y estadísticamente significativa entre el diseño organizacional y el desempeño docente en las instituciones educativas de secundaria UGEL Virú 2020. Dado que el valor del coeficiente rho $=0,566$. Además, el valor de la significancia bilateral $=$ 0,00 es menor que el nivel de significancia de 0,05. Por tanto, a mayor nivel de diseño organizacional mayor nivel de desempeño docente.

7. Se determinó que existe una relación positiva, moderada y estadísticamente significativa entre la gestión instruccional y el desempeño docente en las instituciones educativas de secundaria UGEL Virú 2020. Dado que el valor del coeficiente rho= 0,517. Además, el valor de la significancia bilateral $=0,00$ es menor que el nivel de significancia de 0,05. Por tanto, a mayor nivel de gestión instruccional mayor nivel de desempeño docente (Tabla 24-A).

\section{$\underline{\text { Referencias }}$}

Aldana, J., Salón, M., y Guzmán, N. (2019, junio). Liderazgo sistémico en las competencias gerenciales docentes universitarias. CIENCIAMATRIA. Revista Interdisciplinaria de Humanidades, Educación, Ciencia y Tecnología. (8). Obtenido de http://www.cienciamatriarevista.or g.ve/index.php/cm/article/view/87 169

Banco Mundial (2020). Covid-19: impacto en la educación y respuestas de política pública. Grupo banco mundial.

BID (2020). La educación en tiempos de coronavirus. Los sistemas educativos de América Latina y el Caribe, Covid-2019. Banco Internacional de Desarrollo.

Bolívar, F. (2010). «¿Cómo un liderazgo pedagógico y distribuido mejora los logros académicos?: Revisión de la investigación y propuesta». Magis: Revista Internacional de Investigación en Educación, 3 (5), 79-106 [en línea]. <http://www.javeriana.edu.co/mag is/> [Consulta: 28 enero 2011].

Cattell, R. (1973). Personality and mood by questionnaire. San Francisco: Jossey-Bass.

${ }^{\mathrm{a}}$ Paola Jannet Sánchez Luján; ${ }^{\mathrm{b}}$ Jorge Alberto Asmat Castro y ${ }^{\mathrm{C}}$ María Soledad, Miranda Gerónimo Articulo Protegido por Licencia Creative Commons: BY-NC-ND / Protected by Creative Commons: BY-NC-ND Sendas es una revista de acceso abierto / Sendas is an Open Access Journal. 
Sendas. 1(1), Octubre - Diciembre, 2020.

URL: https://www.revistas.infoc.edu.pe/index.php/sendas

Email: revistasendas@ infoc.edu.pe

De la Cruz, M. (2018). Liderazgo directivo y el desempeño docente en las instituciones educativas de la cuenca Santa Eulalia, 2018. (Tesis de Maestría. Universidad César Vallejo. Perú).

Díaz, A. y Camejo, M. (2015). Epistemología y educación. Uruguay: Espacio Interdisciplinario de la Universidad de la República.

Elmore, R. (2008). Leadership as the practice of improvement. En Pont, B., D. Nusche and D. Hopkins (eds.) (2008). Improving School Leadership, Volume 2 (pp. 37-68). Paris: OECD.

Fischman, D. (2017). El líder transformador I. México: editorial Planeta.

Garay, S. (2019). Liderazgo y logros en las organizaciones escolares de Chile. (Tesis de doctorado, Universidad Complutense de Madrid).

García, I. (2019). Coordinación Pedagógica y Liderazgo distribuido en los Institutos de Secundaria. Universidad de Granada. (Tesis doctoral. Universidad de Granada. España).

García, L. (2009). Nuevos ambientes de aprendizaje. En L. García Arieto,
Vol. 1, $\mathrm{N}^{\circ} 4$, pp. 38 - 64 Diciembre 2020

ISSN $2708-6380$ https://doi.org/10.47192/rcs.v1i4.49

¿Por qué va ganando la educación a distancia? Madrid: UNED.

Garfield, Z., Rueden, C., y Hagen, E. (2018, 22 - 09). The evolutionary anthropology of political leadership. The Leadership Quarterly (30), 59-80. Doi doi.org/10.1016/j.leaqua.

2018.09.001

Gronn, P. (2000). «Distributed properties: A new architecture for leadership». Educational Management \& Administration, 28 (3), 317-338. - (2010). «Leadership: its genealogy, configuration and trajectory». Journal of Educational Administration and History, 42 (4), 405-435

Handy, Ch. (1997); La organización por dentro. Bilbao: edición Deusto.

Leithwood, K. (2009). ¿Cómo liderar nuestras escuelas? Aportes desde la investigación. Santiago de Chile, Chile: Fundación chile.

Luque, R. (2018). Liderazgo directivo y desempeño docente en una institución educativa primaria, Callao 2018. (Tesis de maestría, Universidad César Vallejo, Perú).

Marzano, R., Waters, T., y McNulty, B. (2005). School leadership that works: From research to results.

${ }^{\mathrm{a}}$ Paola Jannet Sánchez Luján; ${ }^{\mathrm{b}}$ Jorge Alberto Asmat Castro y ${ }^{\mathrm{c}}$ María Soledad, Miranda Gerónimo Articulo Protegido por Licencia Creative Commons: BY-NC-ND / Protected by Creative Commons: BY-NC-ND. Sendas es una revista de acceso abierto / Sendas is an Open Access Journal. 
Alexandria, VA: Association for Supervision and Curriculum Development.

Mateo, J. (2000). La evaluación del profesorado y la gestión de la calidad de la educación. Hacia un modelo comprensivo de evaluación sistemática de la docencia, en: Revista de Investigación Educativa, 18 (1), pp.7-34.

McBer, H. (2000). Research into Teacher Effectiveness: A Model of Teacher Effectiveness. London: Department for Education and Employment.

Minedu (2014). Marco de buen desempeño del directivo. Lima: ministerio de educación.

Montenegro, I. (2003). Evaluación del desempeño docente. Bogotá: editorial magisterio.

OCDE (2001). Docentes para las escuelas del mañana. Análisis de los indicadores educativos mundiales. Paris: OECD e Instituto de Estadísticas de la UNESCO.

Ordinola, D. (2019). Liderazgo directivo y desempeño docente en una institución educativa de Tumbes. (Tesis de Maestría. Universidad César Vallejo. Perú).
Padrón, J. (2014): Proyecto de Epistemología en DVD. Doctorado en Ciencias Humanas, Maracaibo: La Universidad del Zulia.

Robinson, V. (2007). School leadership and student outcomes: Identifying what works and why. Inmalee, New South Wales: Australian Council for Educational Leaders.

Salvador, E. y Sánchez, J. (2018). Liderazgo de los directivos y compromiso organizacional Docente. Journal of High Andean Research, 20(1), 115-124. doi://dx.doi.org/10.18271/ria.2018 .335 .

Sánchez, H. y Reyes, C. (1996). Metodología y diseños en la investigación científica. Lima: editorial Mantaro.

Valle, I. (2019). El liderazgo distribuido y la percepción de los equipos directivos en las escuelas públicas de educación primaria madrileñas. (Tesis doctoral. Universidad Pablo de Olavide Sevilla. España).

Waters, T., Marzano, R. J. y McNulty, B. (2003). Balanced Leadership: What 30 years of research tells usabout the effect of leadership on student achievement. Working paper. Denver: McRel.

${ }^{\mathrm{a}}$ Paola Jannet Sánchez Luján; ${ }^{\mathrm{b}}$ Jorge Alberto Asmat Castro y ${ }^{\mathrm{c}}$ María Soledad, Miranda Gerónimo Articulo Protegido por Licencia Creative Commons: BY-NC-ND / Protected by Creative Commons: BY-NC-ND. Sendas es una revista de acceso abierto / Sendas is an Open Access Journal. 
Sendas. 1(1), Octubre - Diciembre, 2020.

URL: https://www.revistas.infoc.edu.pe/index.php/sendas

Email: revistasendas@infoc.edu.pe

Zuñiga, J. (2018). Liderazgo directivo y

desempeño docente en la

Institución Educativa Daniel

Alcides Carrión, San Pablo de
Vol. 1, $\mathrm{N}^{\circ} 4$, pp. 38 - 64 Diciembre 2020

ISSN $2708-6380$ https://doi.org/10.47192/rcs.v1i4.49

Occo, Lircay 2018. (Tesis de Maestría. Universidad César Vallejo. Perú).

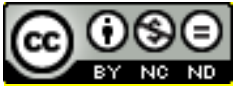

Influencia del Liderazgo directivo en el Desempeño docente,

Virú 2020 (Paola Jannet Sánchez - Luján) Por Revista Sendas se encuentra bajo una Licencia Creative Commons-No Comercial-Sin Derivadas 3.0 Uported.

${ }^{\mathrm{a}}$ Paola Jannet Sánchez Luján; ${ }^{\mathrm{b}}$ Jorge Alberto Asmat Castro y ${ }^{\mathrm{c}}$ María Soledad, Miranda Gerónimo Articulo Protegido por Licencia Creative Commons: BY-NC-ND / Protected by Creative Commons: BY-NC-ND. Sendas es una revista de acceso abierto / Sendas is an Open Access Journal. 